\title{
EDUCAÇÃO À DISTÂNCIA NO ENSINO SUPERIOR: RELATO DE EXPERIÊNCIA EM ESTÁGIO DE DOCÊNCIA
}

\section{DISTANCE EDUCATION IN HIGHER EDUCATION: EXPERIENCE REPORT IN TEACHING INTERNSHIP}

\section{EDUCACIÓN A DISTANCIA EN LA ENSEÑANZA SUPERIOR: INFORME DE EXPERIENCIA EN PRÁCTICAS DE DOCENCIA}

Rebeca Coelho de Moura Angelim¹ ${ }^{1}$, Brígida Maria Gonçalves de Melo Brandão², Verônica Mirelle Alves Oliveira Pereira², Daniela de Aquino Freire ${ }^{3}$, Fátima Maria da Silva Abrão ${ }^{4}$.

\section{RESUMO}

Objetivo: Relatar a experiência de mestrandas em enfermagem na disciplina Estágio de Docência por meio da Plataforma Moodle. Métodos: Trata-se de um estudo descritivo, do tipo relato de experiência onde mestrandas de Enfermagem atuaram como tutoras em um módulo do respectivo curso, utilizando o ensino à distância. Resultados: A tutoria, por meio do ensino à distância, é uma abordagem inovadora que, apesar da separação geográfica existente entre o aluno e o professor, pode promover maior interação entre ambos. Conclusão: Conclui-se que, nessa modalidade, o tutor tem a tarefa de conduzir o processo ensino-aprendizagem, identificando as dificuldades, sanando dúvidas e contribuindo para aumentar o leque de conhecimentos do aluno.

Descritores: Docentes; Educação superior; Educação em enfermagem; Educação à distância.

\begin{abstract}
Objective: To report the experience of master students in nursing attending the subject Teaching Internship through the Moodle Platform. Methods: This is a descriptive study, of experience report kind, in which master students in nursing worked as tutors in a module of the respective course, using distance education. Results: The mentoring in distance learning is an innovative approach that, despite the geographical separation between the student and the professor, can promote greater interaction between both of them. Conclusion: We concluded that, in this modality, the tutor has the task of conducting the teaching-learning process, identifying the difficulties, removing doubts and contributing to increase the student's knowledge.

Descriptors: Faculty; Higher Education; Nursing Education; Distance Education.
\end{abstract}

\section{RESUMEN}

Objetivo: Informar de la experiencia de las alumnas de máster en enfermería en la asignatura de Práctica de Docencia a través de la Plataforma Moodle. Métodos: Se trata de un estudio descriptivo, del tipo relato de experiencia donde alumnas de máster en Enfermería actuaron como tutoras en un módulo del respectivo curso, utilizando la enseñanza a distancia. Resultados: La tutoría, a través de la enseñanza a distancia, es un enfoque innovador, que pese a la separación geográfica existente entre el alumno y el profesor, puede promover una mayor interacción entre ambos. Conclusión: Se concluye que, en esta modalidad, el tutor tiene la tarea de conducir el proceso enseñanza-aprendizaje, identificando las dificultades, aclarando dudas y contribuyendo a aumentar la diversidad de conocimientos del alumno.

Descriptores: Docentes; Educación superior; Educación en enfermería; Educación a distancia.

\footnotetext{
${ }^{1}$ Graduada em Enfermagem. Doutora em Enfermagem pelo Programa Associado de Pós-graduação em Enfermagem da Universidade de Pernambuco e Universidade Estadual da Paraíba. ${ }^{2}$ Graduada em Enfermagem. Mestre em Enfermagem pelo Programa Associado de Pós-graduação em Enfermagem da Universidade de Pernambuco e Universidade Estadual da Paraíba. ${ }^{3}$ Graduada em Enfermagem. Mestranda em Enfermagem pelo Programa Associado de Pós-graduação em Enfermagem da Universidade de Pernambuco e Universidade Estadual da Paraíba. ${ }^{4}$ Graduada em Enfermagem. Doutora em Enfermagem pela Escola de Enfermagem de Ribeirão Preto/USP. Docente na Universidade de Pernambuco.
}

Como citar este artigo:

Angelim RCM, Brandão BMGM, Pereira VMAO, et al. Educação à Distância no Ensino Superior: Relato de Experiência em Estágio de Docência. Revista de Enfermagem do Centro Oeste Mineiro.2019;9:e2672. [Access ]; Available in: DOI: 


\section{INTRODUÇÃO}

Com o passar dos anos, a Enfermagem, no Brasil, veio se consolidando, principalmente após a implantação dos cursos stricto sensu nas décadas de 70 (mestrado) e 80 (doutorado). Os programas existentes fundamentam-se em áreas de concentração voltadas às disciplinas de enfermagem, permitindo que a profissão consolide-se como um campo muito amplo de conhecimento $^{(1)}$.

Tradicionalmente, a formação de docentes para o ensino superior é vista como o preparo de um indivíduo para que este se volte ao conhecimento aprofundado de determinado conteúdo, seja ele prático ou teórico. Como a pós-graduação prioriza a condução de pesquisas, acaba que, erroneamente, pode ser vista apenas como formadora de pesquisadores, ocasionado um menor interesse para a sua função pedagógica, isto é, o seu papel como formadora de professores ${ }^{(2)}$.

Levando em consideração que o conhecimento e a preparação profissional vêm sendo cada vez mais valorizados, inclusive no meio acadêmico, é reconhecida a necessidade de preparação pedagógica dos professores e futuros professores universitários. Nesse contexto, o Estágio de Docência é uma atividade curricular dos programas de pós-graduação stricto sensu que tem o objetivo de capacitar os alunos de mestrado e doutorado para a carreira docente, de acordo com os propósitos da Coordenação de Aperfeiçoamento de Pessoal de Nível Superior $(\mathrm{CAPES})^{(3)}$.

No Programa Associado de Pósgraduação em Enfermagem da Universidade de Pernambuco e Universidade Estadual da Paraíba (PAPGEnf UPE/UEPB), a disciplina Estágio de Docência é obrigatória para todos os alunos e tem duração mínima de seis meses. Nessa disciplina, os alunos ministram aulas em qualquer módulo do curso de Enfermagem da Universidade de Pernambuco (UPE) ou da Universidade Estadual da Paraíba (UEPB), a depender do vínculo dos seus orientadores e sob supervisão dos mesmos.

$O$ atual Projeto Pedagógico do Curso (PPC) de Enfermagem da UPE é organizado em módulos. Cada módulo é semestral e possui estrutura curricular distribuindo os conteúdos nas áreas temáticas que norteiam a formação do profissional enfermeiro. O objetivo é fortalecer a articulação da teoria com a prática, utilizando uma metodologia de participação ativa do aluno $^{(4)}$.

A metodologia ativa estimula a participação dos educandos no processo de construção do próprio conhecimento, utilizando uma abordagem crítica e reflexiva para resolução e avaliação de problemas ${ }^{(5)}$. Em se tratando do PPC do curso de enfermagem e o conceito de metodologia ativa, considera-se que o papel do discente de pós-graduação no processo ensinoaprendizagem, por meio do Estágio de Docência, é um desafio, visto que tem que atuar não somente como moderador, mas também procurar extrair o melhor do aluno, deixando-o ser o protagonista do seu próprio aprendizado.

A prática do Estágio de Docência pode ser realizada em sala aula, de forma presencial, ou por meio de uma plataforma online, na modalidade de Educação à Distância (EAD), que é capaz de complementar o assunto explanado no campo de prática. Algumas das vantagens da EAD é a facilidade ao acesso para aperfeiçoamento, além da possibilidade de poder escolher o melhor momento para estudar ${ }^{(6-7)}$.

Um dos vários ambientes para EAD é a Plataforma Moodle, que se caracteriza como um ambiente em que o tutor elabora um material virtual aos alunos e fica à disposição para contribuir com o aprendizado dos mesmos. Além disso, a plataforma facilita o acesso a diferentes materiais didáticos, além da edição contínua, revisão das mensagens postadas e possibilidade de interação com diferentes pessoas ${ }^{(8)}$.

Com base no que foi exposto e considerando a importância de se realizar o Estágio de Docência, não apenas de forma presencial, mas também à distância, este estudo teve como objetivo relatar a experiência de mestrandas de Enfermagem na disciplina Estágio de Docência por meio da Plataforma Moodle.

\section{MÉTODOS}

Este estudo trata-se de um relato de experiência que emergiu de reflexões de estudantes do curso de mestrado acadêmico, oriundas da experiência na disciplina Estágio de Docência, do PAPGEnf UPE/UEPB.

Para contemplar as atividades do estágio de docência, 8 alunas do mestrado foram escolhidas para desenvolvê-las através da modalidade de EAD, do curso de Bacharel em Enfermagem da UPE, no segundo semestre do ano de 2014 e primeiro semestre de 2015, resultando em uma carga horária de 60 horas por 
semestre e um total de 120 horas. O módulo selecionado pela coordenação do curso de Enfermagem para os dois semestres foi o sexto módulo, no qual havia, em média, 60 alunos. Ao dividir a turma em oito grupos, cada mestrando ficou responsável por 6 ou 7 alunos. A unidade curricular tinha como foco a Saúde do Adulto e a meta de integrar, através de atividades complementares, os assuntos vivenciados em sala de aula e nas práticas assistenciais. As atividades realizadas foram registradas em diários de campo ao longo do semestre.

Para esse relato, serão descritas as experiências vivenciadas por duas mestrandas com os alunos da graduação através da plataforma Moodle de ensino à distância, sob supervisão da coordenação do sexto módulo e dos orientadores da pós-graduação.

\section{RESULTADOS}

Para a inserção dos alunos do curso de Mestrado, inicialmente, foi realizada uma reunião com a coordenadora docente, que expôs todas as orientações acerca da participação e utilização das ferramentas na plataforma Moodle.

As atividades foram realizadas por meio do modelo de tutoria, onde eram elaborados casos clínicos (material didático avaliativo) para os estudantes do curso de Enfermagem do sexto módulo, abrangendo questões direcionadas à Saúde do Adulto.

Os casos clínicos foram organizados em quatro blocos. O primeiro, para fins de simulação e treinamento (nesse caso precisou ser construído apenas um único exemplo de caso clínico), que foi enviado para o professor da graduação responsável pelo recebimento e encaminhamento dos casos para a plataforma. Nesse momento, não havia obrigatoriedade de correção pelo tutor-mestrando, apenas acompanhamento da atividade e orientações quanto às dúvidas encontradas na mesma.

Pensando na necessidade do aluno, a plataforma contemplava um espaço chamado "Fórum tira-dúvidas", onde a interação tutoraluno ocorria de forma online, possibilitando que fossem esclarecidas quaisquer dúvidas sobre as atividades propostas.

Os outros três casos clínicos, elaborados adiante, foram de cunho avaliativo e deveriam ser avaliados pelos mestrandos, definindo uma pontuação que variava de 0 a 10 pontos, de acordo com os critérios pré-estabelecidos (ficha para avaliação das respostas). A nota final adquirida seria agregada à ponderação das provas integradas do módulo.

Os casos clínicos contemplaram assuntos relacionados às patologias clínicas e/ou cirúrgicas, sendo elas voltadas ao sistema respiratório e gástrico, sistema endócrino, hepatectomias e tumores hepáticos, sistema hematológico, sistema tegumentar, sistema imunológico e sistema renal e metabólico. Além disso, contemplaram ainda o processo saúdedoença relacionado a agravos infectocontagiosos e parasitários, como leishmaniose e HIV/Aids.

Os alunos eram divididos em grupos que continham em torno de seis ou sete alunos cada, sob orientação de um tutor-mestrando. Para cada grupo, era determinado o agravo que deveria ser trabalhado.

Destaca-se que, apesar de ser um caso clínico por grupo, a postagem e a avaliação eram individuais e o grupo deveria servir como um suporte para que cada um elaborasse um plano individual de cuidados.

Os casos clínicos eram construídos objetivando os seguintes aspectos: Descrição do caso contendo o relato através da história da doença atual, história médica pregressa, perfil psicossocial, história familiar e informações do exame físico. Assim, era necessário que o aluno tivesse um conhecimento prévio acerca do assunto em questão e/ou buscasse informações cabíveis para definirem o diagnóstico do caso clínico que, por sinal, tinha três opções de resposta.

Para resolução, era solicitado que os alunos definissem o diagnóstico, apontando sinais e sintomas que justificassem o mesmo, inclusive discutindo o porquê da exclusão dos demais diagnósticos. $\mathrm{Na}$ justificativa, o aluno deveria embasar sua discussão clínica com achados da literatura científica recente. E era solicitada a elaboração do Plano de Cuidados de Enfermagem com base na Sistematização da Assistência de Enfermagem (SAE) onde o mesmo deveria apresentar: Diagnósticos de Enfermagem, Intervenções de Enfermagem e Resultados esperados.

Salienta-se que era definido um prazo para resolução dos casos clínicos pelos alunos, assim como tinha um prazo para correção pelos tutores-mestrandos. Todos os tutores deveriam, frequentemente, acessar a plataforma para visualizar possíveis dúvidas e questionamentos dos alunos. 
Por meio das avaliações, foi possível evidenciar que os alunos sentiam dificuldade em elaborar as referências com base nas normas da ABNT (que era uma orientação a seguir) e que as referências citadas, em geral, eram oriundas de livros e manuais. Sendo assim, os tutores viram a necessidade de orientar os alunos quanto às normas da ABNT e estimular a procura de artigos científicos, de preferência dos últimos cinco anos, de modo que os alunos praticassem a pesquisa científica e o interesse pela leitura de artigos.

Vale ressaltar que foi fundamental o feedback do tutor ao escrever seus comentários sobre a avaliação e a nota que foi enquadrada, com comentários contendo pontos positivos e negativos, de modo que 0 aluno pudesse compreender seus erros e acertos para buscar melhorar nas demais atividades.

\section{DISCUSSÃO}

Por meio da disciplina Estágio de docência, percebeu-se que a mesma se propõe a aproximar o mestrando do processo ensino-aprendizagem, sendo esse direcionado para o seu campo de atuação que, no caso, foi o curso de Enfermagem. Proporcionou-se a oportunidade de discutir conteúdos teóricos e aplicá-los à prática supervisionada da docência, seja ela presencial ou à distância.

A educação à distância é considerada uma modalidade de ensino-aprendizagem, apoiada por tecnologias de informação e comunicação, em que o professor e aluno não se encontram na sala de aula convencional, como acontece de costume. Entretanto, tem sido reconhecida como uma modalidade de Educação que exerce um papel importante na sociedade atual ${ }^{(9)}$, visto que expandiu o acesso a conteúdos educacionais somente com a utilização de internet ${ }^{(10)}$. Logo, infere-se que a modalidade EAD é uma forma inovadora de tutoria que transcende a sala de aula, além da possibilidade de acesso a qualquer momento e horário.

Sob o ponto de vista das atividades desenvolvidas, destaca-se, como positivo, a facilidade em esclarecer dúvidas constantes e a aplicabilidade da SAE, tendo em vista que é uma prática assistencial rotineira da enfermagem e, consequentemente, é uma forma de o aluno poder exercer com frequência.

A oportunidade de sanar dúvidas e discutir conteúdos é realizada por meio dos fóruns, em que o tutor possui autonomia para escrever mensagens, fornecer recomendações, lançar perguntas norteadoras, mediar debates e estimular a participação dos alunos. Ou seja, na modalidade EAD, os fóruns são salas de tutoria para fins pedagógicos ${ }^{(11)}$.

Como ponto negativo da modalidade EAD e utilização dos fóruns de discussão neste estudo, destaca-se que alguns alunos queixaram-se do acesso à internet, o que às vezes comprometia a interação na plataforma. Mas, considera-se que isso se caracterizou mais como um transtorno que, rapidamente, era sanado - do que um ponto negativo propriamente dito.

Em relação à prática de estágio de docência na modalidade EAD, ressalta-se que foi uma experiência diferenciada e de muita importância para a formação do futuro professor, tendo em vista que vem sendo algo recorrente na grade curricular dos cursos de graduação, como ferramenta de apoio ao ensino presencial e, por isso, os professores também precisam estar preparados para atuar nessa área.

Em meio a esse contexto, destaca-se que, para o tutor-mestrando, enquanto futuro professor, a modalidade EAD caracteriza-se como mais um modelo de aula a ser vivenciado para a sua formação acadêmica, modelo este que é novo, mas rico em experiências. Já para o aluno, é uma oportunidade de vivenciar um espaço fora do convencional (por transcender a sala de aula), mesmo apresentando algumas semelhanças com o ensino presencial ${ }^{(12)}$.

Em se tratando da experiência de tutoria, vale ressaltar que foi de grande importância, visto que é uma abordagem inovadora que, apesar da separação geográfica existente entre o aluno e o professor, pode promover maior interação entre ambos. Tanto docentes quanto discentes passam a desempenhar um papel mais ativo no processo ensino-aprendizagem, à medida em que interagem virtualmente no decorrer da disciplina/unidade temática.

Esta forma de ensino coloca o aluno como agente do seu processo de aprendizagem e o professor/tutor atua como um agente mediador, sendo importante que este incentive e estimule a participação do aluno ${ }^{(13)}$.

Nessa linha de pensamento, "entende-se que, em um curso na modalidade à distância, o tutor deve adotar uma atitude colaborativa, incentivadora e construtivista, que estimule o aluno e o ajude a desenvolver a curiosidade, a participação e a capacidade de iniciativa" ${ }^{\prime(9)}$. Assim, torna-se relevante instrumentalizar o estudante a "aprender a aprender", de modo que 
os conhecimentos facilitem a construção de uma identidade profissional e as habilidades possam ser transformadas durante a prática profissional ao longo do tempo ${ }^{(14)}$.

Nesse ínterim, tendo em vista que, nos últimos anos, a Enfermagem tem se apresentado como uma ciência em expansão, exigindo dos profissionais maior conhecimento, atualização e aperfeiçoamento $^{(15)}$, vê-se que a sociedade atual necessita de um novo tipo de profissional em todos os setores. Essa necessidade se dá pela busca de competências múltiplas, trabalho em equipe, capacidade de aprender e de adaptar-se a situações novas ${ }^{(16)}$.

Sendo assim, buscando a realização de um cuidado com excelência, faz-se necessário buscar novas modalidades e alternativas de aprendizado que possibilitem, aos estudantes e profissionais da área, uma atualização constante. Uma forma de se alcançar essa demanda crescente na área da Enfermagem é através da incorporação da tecnologia da informação e comunicação em saúde ${ }^{(15)}$.

Faz-se necessário compreender que as Tecnologias de Informação e Comunicação são ferramentas importantes para o sucesso do EAD, desde que não sejam banalizadas como meros instrumentos tecnológicos ${ }^{(17)}$. Dessa forma, é fundamental que todos os atores envolvidos no processo ensino-aprendizagem tenham a consciência de que, geralmente, o sucesso e/ou fracasso do EAD está fortemente ligado às formas como ocorreu a comunicação entre os participantes e os instrumentos tecnológicos da $E A D$, visto que são de suma importância para otimizar a relação tutor-aluno, servindo como uma abordagem eficaz para o engrandecimento dos conhecimentos adquiridos tanto em sala de aula quanto na prática clínica.

O EAD vem democratizando o ensino e proporcionando formação de qualidade e de acordo com a disponibilidade de cada aluno, o que tem contribuído para o aumento da demanda por essa modalidade que, desde o início do século $X X$, tem tido suas metodologias aperfeiçoadas $^{(18)}$. Assim, é uma modalidade que favorece o acesso à informação e à formação do cidadão.

Sendo assim, o EAD é um processo de inclusão e capacitação das tecnologias da informação, que dispõe de diversas vantagens como a eliminação de barreiras de espaço e tempo, economia de recursos financeiros, flexibilidade e acessibilidade ${ }^{(19)}$.
Outro aspecto importante que deve ser mencionado diz respeitos aos fatores de ordem subjetiva, que colaboram para que os alunos, facilmente, se adaptem à tal realidade como a motivação para o estudo, a autoestima, o fato de ser um estudo predominantemente individual, as formas de interação com o tutor, a construção da autonomia e a metacognição ${ }^{(20)}$.

$O$ ato de educar não se dá pelo simples fato de transmitir conhecimentos. Existe, dentro do processo educativo, um ideal, um arcabouço de profissional que quer formar-se e lançar-se no mercado de trabalho. Frente às transformações ocorridas na sociedade como um todo, principalmente nos seus aspectos de relacionamentos interpessoais, valores, crenças e, consequentemente, no processo educativo, percebe-se a necessidade de buscar formas de inovações para alcançar essas transformações. Nesse sentido, a aplicabilidade de mídias educativas sob a modalidade de EAD, atreladas a cursos de capacitação e aperfeiçoamento, contempla a necessidade de profissionais e estudantes (que já estão inseridos no mercado de trabalho e dele não podem se ausentar) buscarem novos conhecimentos ${ }^{(15)}$.

Diante dos aspectos discutidos, cabe ressaltar, então, que a incorporação do EAD significa uma reestruturação do processo ensinoaprendizagem, o qual deve seguir o mesmo rigor científico e metodológico do ensino presencial, cuja contribuição à sociedade tem sua relevância, ao permitir uma flexibilidade para o estudo. Essa, inclusive, é uma característica importante para o indivíduo poder se adequar às demandas da sociedade, dando conta do trabalho e do estudo. Fato, muitas vezes, encontrado na realidade dos indivíduos nos dias atuais.

\section{CONSIDERAÇÕES FINAIS}

O Estágio de Docência constitui-se como uma etapa importante para o aluno de pósgraduação stricto sensu, caracterizando-se como um subsídio de fortalecimento pedagógico no que se refere à preparação para a prática docente.

Dentre os grandes desafios da disciplina Estágio de Docência, está a possibilidade de ter que conduzir o ensino na modalidade à distância. Por meio da experiência de EAD com alunos da graduação, as alunas do mestrado puderam concluir que essa modalidade de ensino requer preparo e estudo, posto que o professor/tutor tem a tarefa de conduzir o processo ensino- 
aprendizagem, identificando as dificuldades, retirando dúvidas e contribuindo para aumentar o leque de conhecimentos do aluno.

Este estudo teve, como limitação, relatar a experiência do Estágio de Docência em apenas uma abordagem, que foi o EAD. Sugere-se que sejam realizados novos relatos de docência em sala de aula e na parte prática, integrando, assim, essas três abordagens e extraindo os pontos positivos e negativos das experiências, como forma de rever o que pode ser melhorado, visando um maior aprendizado para o aluno de graduação e um maior crescimento profissional para mestrando.

\section{REFERÊNCIAS}

1. Robazzi MLCC. O desempenho da pósgraduação stricto sensu em Enfermagem e a busca pela excelência. Rev Latino-Am Enfermagem 2010;18(5):02 telas. DOI: 10.1590/ S0104-11692010000500001

2. Pimentel V, Mota DDCF, Miako K. Reflexões sobre o preparo para a docência na pósgraduação em Enfermagem. Rev Esc Enferm USP. 2007;41(1):161-4. DOI: 10.1590/S0080-62342007 000100022

3. Pachane GG. Políticas de formação pedagógica do professor universitário: Reflexões a partir de uma experiência. Rio de Janeiro: Associação Nacional de Pós-Graduação e Pesquisa em Educação; 2009.

4. Universidade de Pernambuco (UPE). Faculdade de Enfermagem Nossa Senhora das Graças (FENSG). Projeto Pedagógico do Curso. Pernambuco: UPE; 2013.

5. Sebold LF, Martins FE, Rosa R, Carraro TE, Martini JG, Kempfer SS. Metodologias ativas: Uma inovação na disciplina de fundamentos para o cuidado profissional de enfermagem. Cogitare Enferm. 2010 [citado em 7 out 2018]; 15(4):7536. Disponível em: http://revistas.ufpr.br/cogitare/ article/view/20381/13551

6. Mattos LB, Dahmer A, Magalhães CR. Contribuição do curso de especialização em Atenção Primária à Saúde à prática de profissionais da saúde. ABCS Health Sciences 2015; 40(3):184-9. DOI: 10.7322/abcshs.v40i3. 793

7. Pessoni A, Goulart E. Tecnologias e o ensino na área da saúde. ABCS Health Sciences 2015;40(3):

270-5. DOI: 10.7322/abcshs.v40i3.807

8. Ruiz-Moreno L, Leite MT, Ajzen C. Didactic and pedagogic training in health care: cognitive skills developed by postgraduate students in a virtual learning environment. Ciênc Educ. 2013;19(1):217-29. DOI: 10.1590/S151673132013000100015

9. Oliveira ATE, Santos CM, Pereira JA, Fontes LGA, Silva TB, Ferreira PAP. Ferramentas e estratégias de interação e comunicação na prática da tutoria em EAD. Evidência 2017 [citado em 7 out 2018]; 13(13):71-85. Disponível em: http://www.uniaraxa. edu.br/ojs/index.php/evidencia/article/view/532 $\not 509$

10.Saeger MT, Melo AHA. Inserção do egresso de cursos a distância no mercado de trabalho: um estudo voltado para a licenciatura em matemática. EAD em Foco 2016;6(2):155-71. DOI: 10.18264/eadf.v6i2.396

11.Tenório A, Ferrari Júnior J, Tenório T. A visão de tutores sobre o uso de fóruns em cursos a distância. Rev Bras Aprendiz Aberta Distancia 2015 [citado em 7 out 2018]; 14:56-70. Disponível em: http://seer.abed.net.br/edicoes/ 2015/04 A VISÃO DOS TUTORES.pdf

12.Mattos LJG, Santos SC. Os desafios do estágio supervisionado em um curso de licenciatura a distância. EAD em Foco. 2018 [citado em 7 out 2018]; 8(1):e643. Disponível em: http://eademfoco.

cecierj.edu.br/index.php/Revista/article/view/64 $\underline{3 / 286}$

13.Vendruscolo $\mathrm{MI}$, Behar PA. Investigando modelos pedagógicos para educação a distância: Desafios e aspectos emergentes. Educação 2016 [citado em 7 out 2018]; 39(3):302-11. Disponível em: http://www.redalyc.org/html/848/848497 $\underline{92004}$

14.Makuch DMV, Zagonel IPS. Abordagem pedagógica na implementação de programas curriculares na formação do enfermeiro. Esc Anna Nery 2017;21(4):1-9. DOI: 10.1590/2177-9465EAN-2017-0025

15. Matos JC, Lima RRS, Nakata CRG, Castro AF, Guimarães $H C$, Silva AR. A educação a distância no ensino e na prática de enfermagem: Revisão integrativa. REUOL. 2016 [citado em 7 out 2018]; 10(7):2656-68. Disponível em: https://periodicos. ufpe.br/revistas/revistaenfermagem/article/view $\angle 11327 / 13021$

16.Silva AN, Santos AMG, Cortez EA, Cordeiro BC. Limites e possibilidades do ensino à distância (EaD) na educação permanente em saúde: Revisão integrativa. Ciênc Saúde Coletiva 2015;20(4): 1099-107. DOI: 10.1590/1413$\underline{81232015204.17832013}$ 
17.Pons MED, Cardoso Sobrinho J, Remedi JMR. As tecnologias da informação e comunicação e a educação à distância. Rev Eletrôn Gest, Educ Tecnol Ambient. 2013 [citado em 7 out 2018]; 13(13):2661-69. Disponível em: http://road.issn.org/ issn/2236-1170-revistaeletronica-em-gestao-educacao-e-tecnologiaambiental-\#.WjGYgI WnHIU

18.Vianna LJ, Ataide CA, Ferreira MC. Educação a distância no Brasil: Cotidiano, prática, avanços e perspectivas. In: Anais do 110 Encontro Internacional de Formação de Professores e Fórum Permanente de Inovação Educacional, 2015; Aracaju. Aracaju: Unit; 2015. p. 1-11.

19.Fernandes PF. O ideal e o real no perfil de tutores e alunos da educação a distância. Rev Eletrônica Gest Saúde 2012 [citado em 15 jan 2017]; 3(1):1432-39. Disponível em:

http://periodicos.unb.br/index.php/rgs/article/vi ew/14061/9990

20.Oliveira EDSG, Santos L. Tutoria em Educação a Distância: Didática e competências do novo "fazer pedagógico". Rev. Dialogo Educ. 2013; 13(38):203-23. DOI: 10.7213/dialogo.educ. 7642

Nota: Este trabalho fez parte de um relatório da disciplina Estágio de Docência do Curso de Mestrado em Enfermagem do Programa Associado de Pós-Graduação em Enfermagem da Universidade de Pernambuco e Universidade da Paraíba (PAPGEnf UPE/UEPB).

Recebido em: $14 / 12 / 2017$

Aprovado em: 22/10/2018

Endereço de correspondência:

Rebeca Coelho de Moura Angelim

Rua Arnóbio Marquês, 310 - Bairro Santo Amaro

CEP: 50100-130 - Recife/PE - Brasil

E- mail: rebecaangelim@hotmail.com 